\title{
Properties of implanted and CVD incorporated nitrogen-vacancy centers: preferential charge state and preferential orientation
}

\author{
K.-M. C. Fu ${ }^{a, b}$, C. Santori ${ }^{a}$, P. E. Barclay ${ }^{a, c, d}$, A. Faraon ${ }^{a}$, \\ D. J. Twitchen ${ }^{e}$, M. L. Markham ${ }^{e}$ R. G. Beausoleil ${ }^{a}$ \\ ${ }^{a}$ Information and Quantum Systems Lab, Hewlett-Packard Laboratories, 1501 Page Mill Rd., \\ Palo Alto, CA 94304, USA; \\ ${ }^{b}$ Department of Physics, University of Washington, Seattle, Washington 98195, USA; \\ ${ }^{c}$ Institute for Quantum Information Science and Department of Physics and Astronomy, \\ University of Calgary, Calgary, Alberta, Canada; \\ $d$ National Institute for Nanotechnology, Edmonton, Alberta, Canada; \\ e Element Six Ltd., King's Ride Park, Ascot, Berkshire SL5 8BP, UK
}

\begin{abstract}
The combination of the long electron state spin coherence time and the optical coupling of the ground electronic states to an excited state manifold makes the nitrogen-vacancy (NV) center in diamond an attractive candidate for quantum information processing. To date the best spin and optical properties have been found in centers deep within the diamond crystal. For useful devices it will be necessary to engineer NVs with similar properties close to the diamond surface. We report on properties including charge state control and preferential orientation for near surface NVs formed either in CVD growth or through implantation and annealing.
\end{abstract}

\section{INTRODUCTION}

The nitrogen-vacancy (NV) center in diamond is a promising candidate as both a qubit for quantum information processing and a sensor for magnetometry. The promise of this defect lies in the unique spin and optical properties found in centers which were incorporated naturally in mined diamond or accidentally in CVD grown samples. These unintentionally incorporated centers typically lie far from the diamond surface, exhibit the desired negative charge state, exhibit spin coherence times which can exceed a millisecond, ${ }^{1}$ have optical transition linewidths which can approach the lifetime limited value, ${ }^{2,3}$ and exist in all four possible orientations.

Many QIP and magnetic sensing applications, however, will require NV centers to be located extremely close to the diamond surface. For quantum information applications that require an optical bus ${ }^{4,5}$ centers will need to be incorporated into optical microcavities. Cavities based on $\mathrm{GaP}$ on single-crystal diamond, ${ }^{6,7}$ monolithic diamond, ${ }^{8}$ or coupled to centers in nanoparticle diamond ${ }^{9-12}$ will all require centers ranging from 10-100 $\mathrm{nm}$ from the diamond surface. For magnetometry applications the separation between the center and the surface will limit both the device sensitivity and spatial resolution, especially in scanning probe implementations. ${ }^{13-15}$

\begin{tabular}{|c|c|c|c|}
\hline sample name & energy & dose & depth \\
\hline S1 & $10 \mathrm{keV}$ & $1 \mathrm{e} 11 \mathrm{~cm}^{-2}$ & $14 \pm 5 \mathrm{~nm}$ \\
S2 & $50 \mathrm{keV}$ & $1 \mathrm{e} 11 \mathrm{~cm}^{-2}$ & $62 \pm 14 \mathrm{~nm}$ \\
\hline
\end{tabular}

Table 1. Implantation conditions for the two N-implanted samples studied. Implantation depths are calculated using SRIM software ${ }^{16}$

Some applications, for instance ensemble-based magnetometry and QIP based on cavity-coupled NV centers, require a specific orientation of the NV center. In these cases it would be preferential to select only one of the four possible orientations. In this paper we discuss two advancements in NV engineering. First we demonstrate control over the NV charge state for NV centers created by implantation and annealing. Second we show control over the NV orientation for NVs incorporated in CVD diamond grown on a $\langle 110\rangle$ substrate.

Advances in Photonics of Quantum Computing, Memory, and Communication IV, edited by Zameer UI Hasan, Philip R. Hemmer, Hwang Lee, Charles M. Santori, Proc. of SPIE Vol. 7948, 79480S · C 2011 SPIE · CCC code: 0277-786X/11/\$18 · doi: 10.1117/12.876169

Proc. of SPIE Vol. 7948 79480S-1 


\section{CONTROL OF CHARGE STATE OF NV CENTERS FORMED BY IMPLANTATION AND ANNEALING}

One common technique for creating near-surface NV centers is by nitrogen ion implantation and high temperature annealing. ${ }^{17}$ During the implantation process vacancies are created. During the annealing vacancies diffuse and some fraction of the vacancies combine with the implanted nitrogen to form NV centers. This method can be used to create centers only nanometers away from the diamond surface. In low nitrogen samples however this techniques produces NV centers in the undesired neutral charge state $\mathrm{NV}^{0} .{ }^{18}$ Here we show that by subsequently annealing the sample in an oxygen atmosphere the charge state can be converted to the desired negatively charged state $\mathrm{NV}^{-} .{ }^{19} \mathrm{NV}^{-}$centers convert back to the neutral state when exposed to a hydrogen plasma and can be converted back again to $\mathrm{NV}^{-}$with a second oxygen anneal.
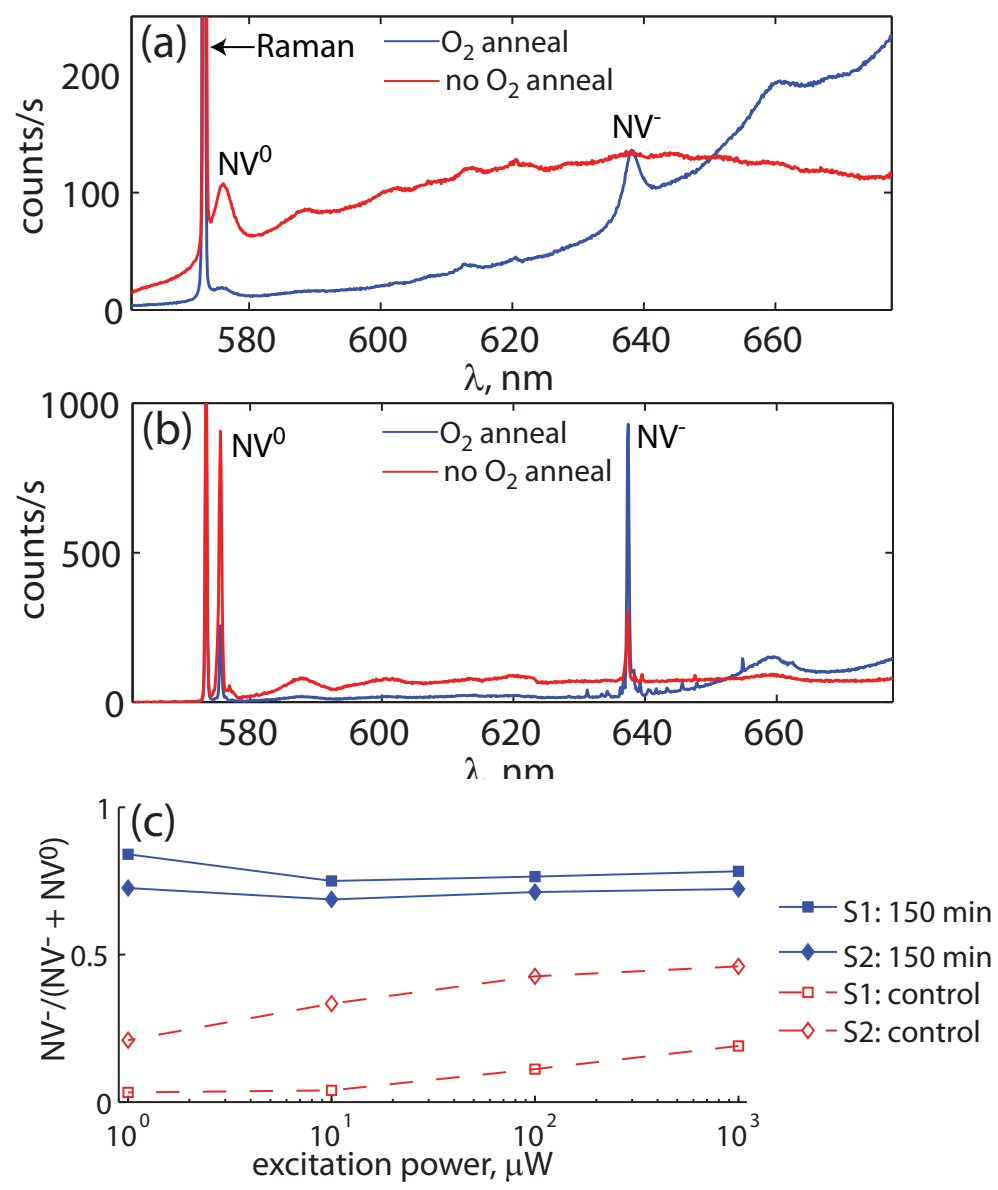

Figure 1. a) Room temperature PL spectra of sample S1 in the same implantation region before and after 150 minutes of $\mathrm{O}_{2}$ annealing. $1 \mathrm{~mW}$ excitation power. b) $10 \mathrm{~K} \mathrm{PL}$ spectra of sample S1. $1 \mathrm{~mW}$ excitation power. c) The ratio of the $\mathrm{NV}^{-}$ZPL intensity to the total ZPL intensity as a function of optical excitation power at $10 \mathrm{~K}$.

NV centers were created in two samples by nitrogen implantation (CORE Systems) through a TEM grid mask and subsequently annealed at $900{ }^{\circ} \mathrm{C}$ for 1 hour in a $\mathrm{H}_{2} / \mathrm{Ar}$ atmosphere. Implantation conditions and depths are given in Table 1 . Half of the sample surface was subsequently masked by $125 \mathrm{~nm}$ of $\mathrm{SiO}_{2}$ deposited by electron beam evaporation. Photoluminescence spectra of the neutral $\mathrm{NV}^{0}$ and negatively charged $\mathrm{NV}^{-}$in Fig. 1a,b show that the predominate NV charge state after implantation and annealing is the undesired $\mathrm{NV}^{0}$. Previous work found this result consistent with the existence of an acceptor level near the diamond surface. ${ }^{18}$ One possible acceptor candidate is $s p^{2}$-hybridized carbon created during the implantation process. ${ }^{20}$ Previous work in diamond nanoparticles has shown that the selective removal of $s p^{2}$-hybridized (graphite-like) carbon over 
$s p^{3}$-hybridized (diamond-like) carbon can be achieved by annealing the sample in air at elevated temperatures. Using the same technique we anneal the three substrates for 150 minutes at $465{ }^{\circ} \mathrm{C}$. As shown in Fig. 1a,b the predominate charge state after the oxygen anneal is $\mathrm{NV}^{-}$.

The ratio of the $\mathrm{NV}^{-}$to $\mathrm{NV}^{0}$ line can be dependent on the optical excitation power. ${ }^{21}$ This "photochromatic" effect is attributed to optically-induced charge state transfer from nearby impurities or charge traps to the NV center. We measure the ratio of the $\mathrm{NV}^{-}$zero phonon line (ZPL) intensity to the sum of the $\mathrm{NV}^{-}$and $\mathrm{NV}^{0} \mathrm{ZPL}$ intensities as a function of excitation power as shown in Fig. 1c. In the masked region of the sample, which was not exposed to the oxygen atmosphere, the photochromatic effect is observed. As the optical power is decreased the $\mathrm{NV}^{-}$component decreases suggesting that the predominate charge state is $\mathrm{NV}^{0}$ in the absence of optical excitation. In contrast, in the unmasked region the ratio is relatively independent of optical excitation power. This result indicates that for oxygen treated samples the predominate charge state is $\mathrm{NV}^{-}$in the dark.
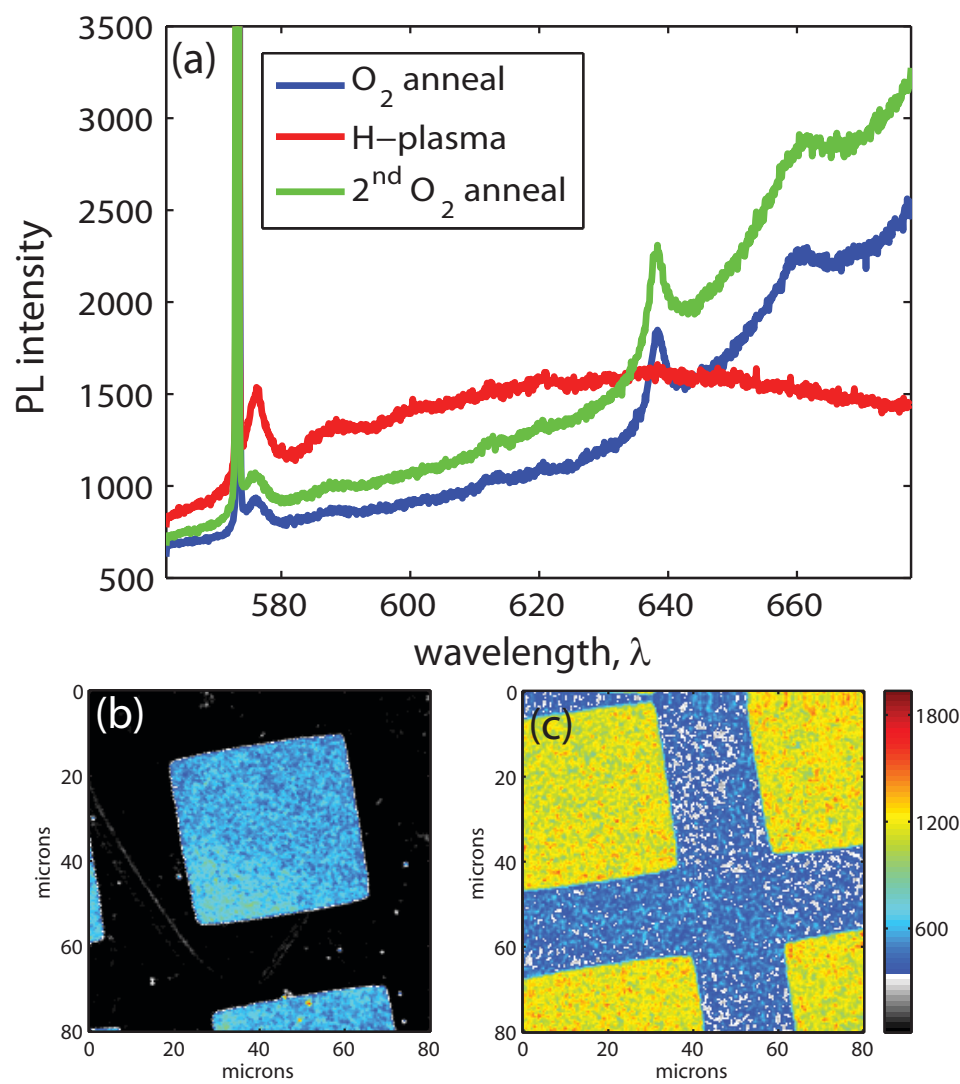

Figure 2. a) Photoluminescence spectra before the h-plasma, after the h-plasma, and after the second oxygen anneal. The absolute intensities between spectra taken before or after the h-plasma cannot be compared due to possible changes in the microscope.b) Confocal image of NV implantation squares defined by the TEM grid after exposure to a hydrogen plasma. Sample was previously implanted with $50 \mathrm{keV} \mathrm{N} \mathrm{N}^{+}$ions at a dose of $1 \mathrm{e} 11 \mathrm{~cm}^{-2}$. c) Confocal image of the same sample after a subsequent oxygen anneal.

In order to demonstrate full control of the charge state it is necessary to demonstrate conversion back to $\mathrm{NV}^{0}$. An alternative to inducing more implantation damage is to hydrogen terminate the surface. A hydrogen terminated diamond surface exhibits a p-type surface conductive layer. ${ }^{22}$ In order to create an h-terminated surface we expose an oxygen-terminated diamond surface to a hydrogen plasma. ${ }^{20}$ Photoluminescence spectra shown in Fig. 2a show that the charge state is $\mathrm{NV}^{0}$ after exposure to the hydrogen plasma. To test the reversibility of this conversion we next anneal the sample in a second oxygen anneal. As expected and shown in Fig. 2a, the charge state after the second oxygen anneal returns to $\mathrm{NV}^{-}$. 
Confocal images of the implantation regions before and after the second oxygen anneal obtained with $532 \mathrm{~nm}$ excitation and 650-800 nm collection are shown in Fig. 2b,c. After the oxygen anneal a marked increase in the photoluminescence intensity is observed in not only the implantation region but also the masked regions. PL spectra confirm that the PL between the squares is predominantly $\mathrm{NV}^{-}$. Further investigations are required to determine the origin of these newly created centers.

\section{PREFERENTIAL ORIENTATION OF NV CENTERS GROWN ON A $\langle 110\rangle$ SURFACE}

In addition to charge state, a second characteristic which may be desirable to control is the NV orientation. In the diamond lattice the NV center symmetry axis can point along any one of the four $\langle 111\rangle$ crystallographic axes. For QIP applications the orientation of the NV optical dipole will be important for NV-cavity coupling. For magnetometry applications the highest sensitivity magnetometers will be obtained in ensemble systems in which all NVs are similarly oriented. Preferential orientations of NV centers created by implantation and annealing has not been demonstrated. Here we show that control over the NV orientation is possible however for NVs created during the CVD diamond growth process on a $\langle 110\rangle$ surface. The four possible NV orientations are shown in

Fig. 3. For two of the four orientations the NV axis lie in the $\langle 110\rangle$ growth plane. The incorporation of these types of centers is suppressed in $\langle 110\rangle$ growth.

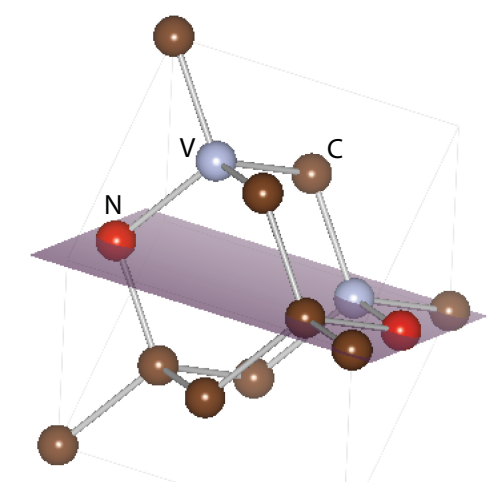

Figure 3. A diamond unit cell. Shaded plane is $\langle 110\rangle$. One of the two in-plane and one of the two out-of-plane NV orientations are depicted. Incorporation of in-plane orientations is almost completely suppressed during $\langle 110\rangle$ growth.

We study a high-purity epitaxial diamond layer grown on a $\langle 110\rangle$ oriented diamond substrate. Single NV centers could be resolved in scanning confocal images. In order to determine NV orientation, images were obtained for nine different linear excitation polarizations. If the surface normal is defined as [110], the linear polarization is varied from 0 to 160 degrees in equal steps with polarization angle 0 corresponding to [001]. As discussed in Ref.[23], the excitation polarization dependence can be used to distinguish between different NV orientations.

Two $75 \times 75 \mu \mathrm{m}$ confocal images taken $130 \mu \mathrm{m}$ beneath the diamond surface are shown in Fig. 4 . Fig. $4 \mathrm{a}$ shows the average intensity from the spatially resolved NVs. Variances in intensity can be due to different spatial depths as well as different NV orientations. Fig. 4b is a composite image of the 9 confocal scans in which laser polarization is encoded into three color channels (magenta- 0 degrees, yellow-60 degrees, and cyan-120 degrees) according to Ref.[23]. In the entire image only one center has a different polarization dependence. A zoomed in image of this defect is shown in Fig. 4c.

In Fig. 5a the polarization intensity dependence of six NVs of the preferred orientation is shown. Variation in overall brightness may be due to mulitple NVs or NVs being out of focus. All NVs show the same polarization dependence. The calculated polarization dependence for both in-plane and out-of-plane NVs is shown in Fig. 5b. It is possible to distinguish between the two in-plane centers which have a markedly different polarization 


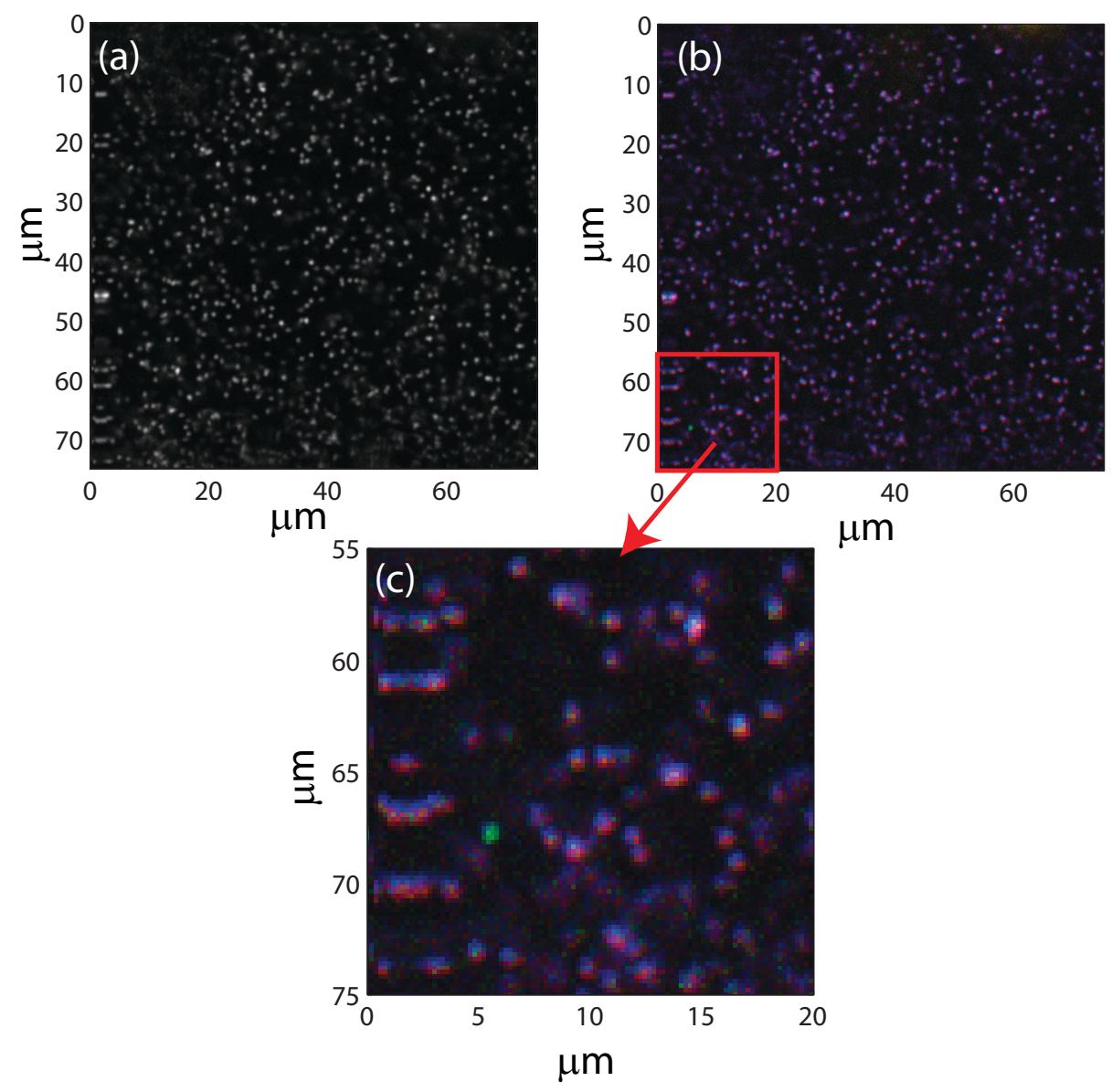

Figure 4. Confocal images of single NV centers in CVD layer. a) Grey-scale intensity sum of all polarization images. b) Polarization encoded composite image. c) Zoom-in image of single out-of-plane NV.

dependence than the out-of-plane centers. The contrast observed in the experimental curves, $\left(I_{\max }-I_{\min }\right) / I_{\max }$ in which $I_{\max (\min )}$ is the maximum (minimum) intensity in the polarization curve, varies from 0.32-0.36 which is consistent with the theoretical contrast of 0.33 .

Reducing the number of NV orientations from 4 orientations to 2 orientations is a significant advancement and should lead to increased optically-detected magnetic resonance contrast and thus increased magnetic sensitivity in ensemble-based magnetometry. In this particular sample we are able to observe NVs at the surface within the optical depth of focus (2-3 microns). Future directions for this work include creating preferentially oriented NVs very close to the diamond surface (10-100 nm) and suppressing the excitation of one of the two orientations through a combination of the optical excitation polarization and diamond orientation.

\section{CONCLUSIONS}

Based on the optical and spin properties of centers deep within the diamond lattice, NV centers hold a lot of promise for applications in quantum information processing and magnetometry. Now one of the focuses of advancing this field is creating NVs intentionally, near the surface, and with specific properties (charge state, orientation) in order to engineer practical devices. In the first part of this paper we showed charge state control between the $\mathrm{NV}^{-}$and $\mathrm{NV}^{0}$ states for NVs formed near the diamond surface by ion implantation and annealing. In the second part of the paper we showed preferential orientation of NV centers incorporated in CVD diamond grown on a $\langle 110\rangle$ substrate. 

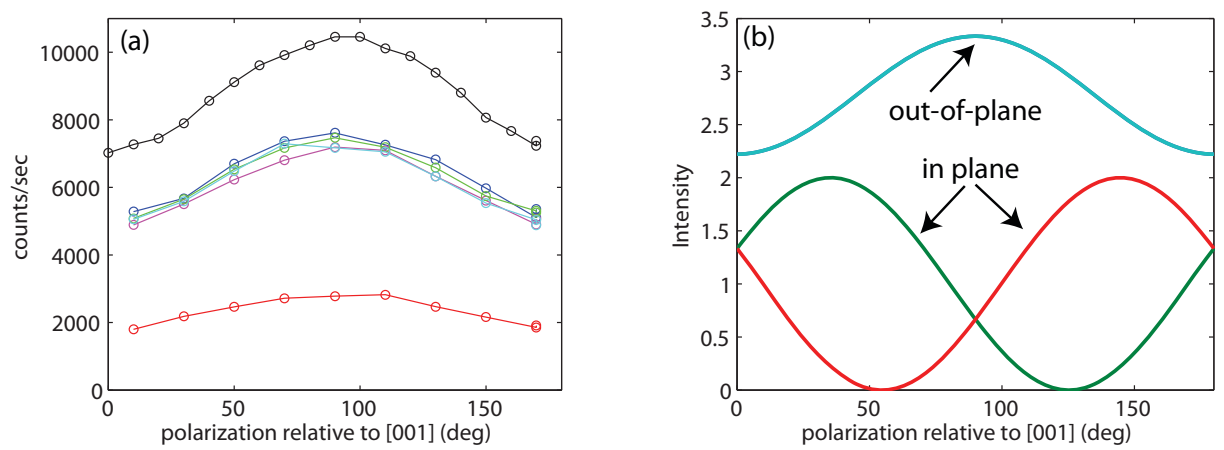

Figure 5. (a) Polarization dependence of the PL intensity for 6 NVs. (b) Theoretical polarization dependence for in-plane and out-of-plane NVs. Model does not include saturation effects.

\section{ACKNOWLEDGEMENTS}

This material is based upon work supported by the Defense Advanced Research Projects Agency under Award No. HR0011-09-1-0006.

\section{REFERENCES}

1. G. Balasubramanian, P. Neumann, D. Twitchen, M. Markham, R. Kolesov, N. Mizuochi, J. Isoya, J. Achard, J. Beck, J. Tissler, V. Jacques, P. R. Hemmer, F. Jelezko, and J. Wrachtrup, "Ultralong spin coherence time in isotopically engineered diamond," Nature Mater. 8, pp. 383-387, May 2009.

2. K.-M. C. Fu, C. Santori, P. E. Barclay, L. J. Rogers, N. B. Manson, and R. G. Beausoleil, "Observation of the dynamic jahn-teller effect in the excited states of nitrogen-vacancy centers in diamond," Phys. Rev. Lett. 103(25), p. 256404, 2009.

3. A. Batalov, C. Zierl, T. Gaebel, P. Neumann, I.-Y. Chan, G. Balasubramanian, P. R. Hemmer, F. Jelezko, and J. Wrachtrup, "Temporal coherence of photons emitted by single nitrogen-vacancy defect centers in diamond using optical rabi-oscillations," Phys. Rev. Lett. 100(7), p. 077401, 2008.

4. L. Childress, J. M. Taylor, A. S. Sorensen, and M. D. Lukin, "Fault-tolerant quantum repeaters with minimal physical resources and implementations based on single-photon emitters," Phys. Rev. A 72, p. 52330, 2005.

5. P. van Loock, T. D. Ladd, K. Sanaka, F. Yamaguchi, K. Nemoto, W. J. Munro, and Y. Yamamoto, "Hybrid quantum repeater using bright coherent light," Phys. Rev. Lett. 96, p. 240501, 2006.

6. K.-M. C. Fu, C. Santori, P. E. Barclay, I. Aharonovich, S. Prawer, N. Meyer, A. M. Holm, and R. G. Beausoleil, "Coupling of nitrogen-vacancy centers in diamond to a gap waveguide," Appl. Phys. Lett. 93(23), p. 234107, 2008.

7. P. E. Barclay, K.-M. C. Fu, C. Santori, and R. G. Beausoleil, "Chip-based microcavities coupled to nitrogenvacancy centers in single crystal diamond," Appl. Phys. Lett. 95(19), p. 191115, 2009.

8. A. Faraon, P. Barclay, C. Santori, K.-M. Fu, and R. Beausoleil, "Resonant enhancement of the zero-phonon emission from a color center ina diamond cavity." http://arxiv.org/abs/1012.3815, 2010.

9. P. E. Barclay, C. Santori, K.-M. Fu, R. G. Beausoleil, and O. Painter, "Coherent interference effects in a nano-assembled diamond nv center cavity-qed system," Opt. Express 17(10), pp. 8081-8097, 2009.

10. J. Wolters, A. W. Schell, G.Kewes, N. Nusse, M. Schoengen, and H. Doscher, "Enhancement of the zero phonon line emission from a single nitrogen vacancy center in a nanodiamond via coupling to a photonic crystal cavity," Appl. Phys. Lett. 97, p. 141108, 2010.

11. Y.-S. Park, A. K. Cook, and H. Wang, "Cavity qed with diamond nanocrystals and silica microspheres," Nano Lett. 6(9), pp. 2075-2079, 2006.

12. D. Englund, B. Shields, K. Rivoire, F. Hatami, J. Vuckovic, H. Park, and M. D. Lukin, "Deterministic coupling of a single nitrogen vacancy center to a photonic crystal cavity," Nanoletters 10, p. 3922, 2010.

13. C. L. Degen, "Scanning magnetic field microscope with a diamond single-spin sensor," Appl. Phys. Lett. 92, p. 243111, 2008. 
14. G. Balasubramanian, I. Y. Chan, R. Kolesov, M. Al-Hmoud, J. Tisler, C. Shin, C. Kim, A. Wojcik, P. R. Hemmer, A. Krueger, T. Hanke, A. Leitenstorfer, R. Bratschitsch, F. Jelezko, and J. Wrachtrup, "Nanoscale imaging magnetometry with diamond spins under ambient conditions," Nature 455, pp. 648-651, Oct. 2008.

15. J. R. Maze, P. L. Stanwix, J. S. Hodges, S. Hong, J. M. Taylor, P. Cappellaro, L. Jiang, M. V. G. Dutt, E. Togan, A. S. Zibrov, A. Yacoby, R. L. Walsworth, and M. D. Lukin, "Nanoscale magnetic sensing with an individual electronic spin in diamond," Nature 455, pp. 644-647, Oct. 2008.

16. J. Zeigler, "The stopping range of ions in matter, srim-2008," 2008.

17. G. Davies and M. F. Hamer, "Optical studies of the $1.945 \mathrm{eV}$ vibronic band in diamond," Proc. R. Soc. Lond. A 348, pp. 285-298, 1976.

18. C. Santori, P. E. Barclay, K.-M. C. Fu, and R. G. Beausoleil, "Vertical distribution of nitrogen-vacancy centers in diamond formed by ion implantation and annealing," Phys. Rev. B 79(12), p. $125313,2009$.

19. K.-M. C. Fu, C. Santori, P. E. Barclay, and R. G. Beausoleil, "Conversion of neutral nitrogen-vacancy centers to negatively charged nitrogen-vacancy centers through selective oxidation," Appl. Phys. Lett. 96(12), p. 121907, 2010.

20. J. Ristein, "Electronic properties of diamond surfaces-blessing or curse for devices?," Diamond EG Rel. Mat. 9(3-6), pp. 1129-1137, 2000.

21. T. Gaebel, M. Domhan, C. Wittmann, I. Popa, F. Jelezko, J. Rabeau, A. Greentree, S. Prawer, E. Trajkov, P. Hemmer, and J. Wrachtrup, "Photochromism in single nitrogen-vacancy defect in diamond," Appl. Phys. B 82, p. 243, 2006.

22. F. Maier, M. Riedel, B. Mantal, J. Ristein, and L. Ley, "Origin of surface conductivity in diamond," Phys. Rev. Lett. 85, p. 3472, 2000. 